\title{
Resistance to extinction as a function of amount of reinforcement and reinforcement schedule '
}

\author{
CHARLES I. BROOKS ${ }^{2}$ AND ROBERT H. DUFORT \\ WAKE FOREST UNIVERSITY, NORTH CAROLINA
}

Sixty rats were run in an experimental design that factorially combined large and small amounts of reinforcement with $0,12,24$, and 56 partial-reinforcement trials given after 28 continuous-reinforcement trials in a runway. Resistance to extinction was an increasing function of number of partial-reinforcement trials under large reinforcement; under small reinforcement, the partial-reinforcement groups did not differ in extinction. The results were discussed within the framework of frustration theory.

When continuous reinforcement (CR) is interpolated between partial reinforcement (PR) and extinction (PR-CR) in a runway, the partial reinforcement effect is obtained (Theios, 1962). Donin, Surridge, \& Amsel (1967) have shown that this same effect is obtained when immediate reinforcement trials are interpolated between a series of partial delay-of-reinforcement trials and extinction. These findings can be interpreted (Deese \& Hulse, 1967; Donin et al, 1967) in terms of Amsel's (1958, 1962) frustration theory.

Recently, Sutherland, Mackintosh, \& Wolfe (1965) reported that when $P R$ is interpolated between $C R$ and extinction (CR-PR), resistance to extinction (Rn) is greater than that found with a CR-only condition, yet less than that found with either a PR-only condition or a PR-CR condition. Although Hothersall (1966) viewed the findings of the Sutherland et al experiment as incompatible with frustration theory, others (Theios \& McGinnis, 1967) have expressed the belief that the CR-PR paradigm can yield results that are.consistent with this theory. The present experiment attempts to extend the analysis of the CR-PR paradigm in terms of frustration theory. The theory suggests that CR and PR are the lower and upper limits, respectively, for the conditioning of frustration cues to the instrumental runway response. Since the conditioning of these cues to running is assumed to directly control $\mathrm{Rn}$, it follows that $\mathrm{Rn}$ should increase as the number of PR trials given after CR training is increased. Furthermore, since the motivational intensity of frustration is assumed to increase as incentive increases, the effect on $\mathrm{Rn}$ of increasing the number of $\mathrm{PR}$ trials after CR should be greater under a large reinforcement conditions (LA) than under a small reinforcement condition (SM). The design factorially combined LA and SM with $0,12,24$, and $56 \mathrm{PR}$ trials given after $28 \mathrm{CR}$ trials. The 0-PR condition represents CR-only training; frustration theory predicts, and studies (e.g., Hulse, 1958; Wagner, 1961) have confirmed, that SM will show greater Rn than LA. As PR training is increased, $\mathrm{Rn}$ is expected to increase, and this effect should appear more readily under LA than SM. This analysis, then, leads to the expectation of an interaction between reinforcement and $\mathrm{PR}$ conditions with regard to the Rn measure.

Method

The Ss were 64 male albino rats of the Holtzman strain, 93 days old at the beginning of the adaptation period of the experiment. Elght Ss were randomly assigned to each of the eight groups formed by the factorial combination of LA (10 .045 $\mathrm{g}$ Noyes pellets) vs SM (1 .045 $\mathrm{g}$ Noyes pellet) and number of PR trials $(0,12,24$, and 56) following CR training. Two Ss were lost from the SM, 12-PR group; to maintain proportionality, two randomly selected $\mathrm{Ss}$ were discarded from the LA, 12-PR group.

The apparatus was a straight unpainted wooden runway, 4 in. wide, with a 12 in. start box, a.48 in. alley, and a 12 in. goal box. Sliding doors separated the runway into three sections. Photocell circuits provided measures of starting and running time. Starting time was measured from the opening of the start box door to a beam located 6 in. from the door. Running time was measured from the first beam to a second beam 18 in. from the end of the goal box.

Ten days prior to acquisition, the adaptation period started. Ss were placed on a $23 \mathrm{~h}$ food deprivation schedule, with free access to water but only $1 \mathrm{~h}$ access to food each day. Ss were handled daily and were allowed to explore the runway individually for $5 \mathrm{~min}$ on each of the last three adaptation days. All Ss then received $28 \mathrm{CR}$ trials. On Day 1 of CR acquisition, each $\mathrm{S}$ was given one trial. On Day 2, each $\mathrm{S}$ received three trials. Four trials a day were given thereafter. An intertrial interval of $10 \mathrm{~min}$ was used throughout the experiment; during this interval, $S$ remained in a compartment of a carrying case. Following $28 \mathrm{CR}$ trials, the 0-PR groups started extinction while the remaining groups received the appropriate amount of PR acquisition prior to extinction. The PR Ss received two reinforced (R) and two nonreinforced (N) trials each day. On $R$ trials, $S$ was removed from the box after eating, and on $\mathrm{N}$ trials, $\mathrm{S}$ was removed after a $20 \mathrm{sec}$ confinement. All groups received four extinction trials a day for 10 days. On each trial, $S$ was removed after $20 \mathrm{sec}$ in the goal box.

\section{Results and Discussion}

Starting and running times were transformed to speeds in $\mathrm{ft} / \mathrm{sec}$; only the running speed measure is reported. The main interest involves performance during extinction. Since acquisition data showed a con- 


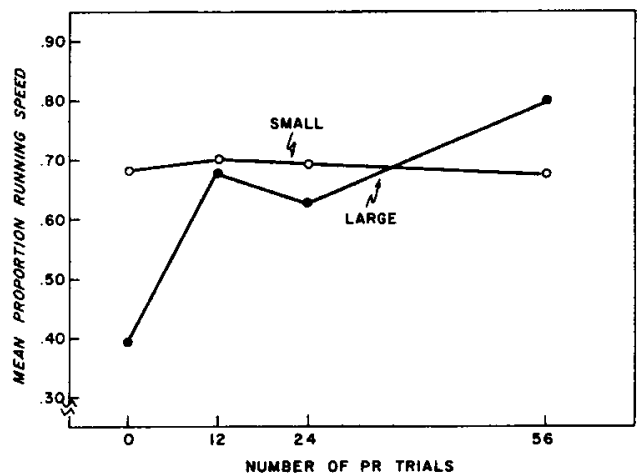

Fig. 1. Runnirg speed in extinction as a proportion of terminal acquisition performance.

sistent superiority of LA over SM for all PR conditions, extinction speed for each $S$, for each trial, was expressed as a proportion of the mean speed $S$ had reached over the last four acquisition trials and the first extinction trial. Figure 1 presents mean proportion running speed for 39 extinction trials. Analysis of variance yielded a significant reinforcement by $P R$ interaction $(F=6.89$, df $=3 / 52, p<.001)$. The nature of the interaction was evaluated by individual group comparisons. For 0 PR, LA showed less Rn than SM ( $<<.001)$, while for 56 PR, LA showed greater $R$ n than $S M(p<.025)$. For 12 and $24 \mathrm{PR}$, no difference between reinforcement condittons was found $(p>.05)$. For $S M$, there were no differences between any of the PR groups ( $p>.05)$. For LA, 0 PR showed less $R$ n than each of the other groups ( $p<.01)$; the 12- and 24-PR groups did not differ ( $p>.05)$; and 56 PR showed greater $R n$ than 24 PR $(p<.01)$.

The data thus showed the expected interaction between reinforcement and PR conditions. However, failure to find variations in the SM groups as a function of PR trials was not anticipated, although it has been noted (Black \& Spence, 1965) that effects of PR variations are not easily demonstrated with small reinforcement. Strict interpretation of the increasing $R n$ shown by the LA groups as reflecting the conditioning of frustration cues to running would be aided by Rn measures on CR-only control groups given the same total numbers of acquisition trials. It seems well established, however, that $\mathrm{Rn}$ decreases with increasing numbers of $\mathrm{CR}$ trials and increasing amounts of reinforcement (e.g., Armus, 1959; Ison \& Cook, 1964). It is concluded that the results under LA reflect the development of frustration and that the CR-PR paradigm offers a useful means of studying this process, at least under conditions of relatively large incentive.

\section{References}

AMSEL, A. The role of frustrative non-reward in noncontinuous reward situations. Psychol Bull, 1958, 55, 102-119.

AMSEL, A. Frustrative nonreward in partial reinforcement and discrimination learning: some recent history and a theoretical extension. Psychol Rev., 1962, 69, 306-328.

ARMUS, H. L. Effect of magnitude of reinforcement on acquisition and extinction of a running response. $J$. exp. Psychol, 1959, 58, 61-63.

BLACK, R. W., \& SPENCE, K. W. Effects of intertrial reinforcement on resistance to extinction following extended training. J. exp. Psychol, $1965,70,559-563$.

DEESE, J., \& HULSE, S. H. The psychology of learning. New York: McGraw-Hill, 1967.

DONIN, J. A., SURRIDGE, C. T., \& AMSEL, A. Extinction following partial delay of reward with immediate continuous reward interpolated, at 24hour intertrial intervals. $J$. exp. Psychol, 1967, 74, $50-53$.

HOTHERSALL, D. Resistance to extinction when continuous reinforcement is followed by partial reinforcement. J. exp. Psychol., 1966, 72, 109-112.

HULSE, S. H. Amount and percentage of reinforcement and duration of goal confinement in conditioning and extinction. J. exp. Psychol, $1958,56,48-57$.

ISON, J. R., \& COOK, P. E. Extinction performance as a function of incentive magnitude and number of acquisition trials. Psychon. Sci, $1964,1,245-246$.

SUTHERLAND, N. S., MACKINTOSH, N. J., \& WOLFE, J. B. Extinction as a function of the order of partial and consistent reinforcement. J. exp. Psychol, 1965, 69, 56-59.

THEIOS, J. The partial reinforcement effect sustained through blocks of continuous reinforcement. J. exp. Psychol, 1962, 64, 1-6.

THEIOS, J., \& MoGINNIS, R. W. Partial reinforcement before and after continuous reinforcement. J. exp. Psychol, 1967, 73, 479-481.

WAGNER, A. R. Effects of amount and percentage of reinforcement and number of acquisition trials on conditioning and extinction. J. exp. Psychol, 1961, 62, 234-242.

\section{Notes}

1. The data reported are from a M.A. thesis performed by the senior author under the supervision of the junior author. Publication of this research was supported by a grant to the junior author from the Graduate Council of Wake Forest University.

2. Now at Syracuse University. 